Artículo

\title{
Disminución de las horas frío como efecto del cambio climático en México
}

\author{
Guillermo Medina-García ${ }^{1 \S}$ \\ José Grageda-Grageda ${ }^{2}$ \\ José Ariel Ruiz-Corral ${ }^{3}$ \\ José Israel Casas-Flores ${ }^{1}$ \\ Víctor Manuel Rodríguez-Moreno ${ }^{4}$ \\ Celia de la Mora-Orozco 5
}

${ }^{1}$ Campo Experimental Zacatecas-INIFAP. Carretera Zacatecas-Fresnillo km 24.5, Calera, Zacatecas. CP. 98500. Tel. 01(800) 0882222, ext. 82306. (casas.israel@inifap.gob.mx). ${ }^{2}$ Campo Experimental Costa de Hermosillo-INIFAP. Pascual Encinas Félix núm. 72, Col. La Manga, Hermosillo, Sonora. CP. 83220. Tel. 01(800) 0882222, ext. 81322. (grageda.jose@ inifap.gob.mx). ${ }^{3}$ Centro Universitario de Ciencias Biológicas y Agropecuarias-Universidad de Guadalajara. Camino Ing. Ramón Padilla Sánchez núm. 2100, Predio Las Agujas, Nextipac, Zapopan, Jalisco. CP. 45510. (arielcajeme@ hotmail.com). ${ }^{4}$ Campo Experimental Pabellón-INIFAP. Carretera Aguascalientes-Zacatecas km 32.5, Pabellón de Arteaga, Aguascalientes. CP. 20660. Tel. 01(800) 0882222, ext. 82525. (rodriguez.victor@ inifap.gob.mx). ${ }^{5}$ Campo Experimental CentroAltos de Jalisco-INIFAP. Carretera Tepatitlán-Lagos de Moreno km 8, Tepatitlán de Morelos, Jalisco. CP. 47600. Tel. 01(800) 0882222, ext. 84503. (delamora.celia@inifap.gob.mx).

${ }^{\S}$ Autor para correspondencia: medina.guillermo@inifap.gob.mx.

\section{Resumen}

En México los frutales caducifolios se cultivan en el centro y norte del país donde la temperatura, al final del otoño y durante el invierno, es baja para que ocurra una defoliación de los árboles y presenten un letargo durante el invierno. El calentamiento global, como efecto del cambio climático, tiene el potencial de reducir el frío disponible en el invierno y afectar la producción de los árboles frutales caducifolios. El objetivo de este trabajo fue conocer el efecto del cambio climático, sobre la acumulación de horas frío en el periodo de invierno. Se realizó un análisis histórico sobre la variación del número de horas frío (HF) y un análisis similar en los escenarios climáticos 2030, 2050 y 2070 en los RCP 4.5 y 8.5. Tanto en el análisis histórico como en los escenarios se encontraron efectos del cambio climático, el cual será desfavorable para los frutales de clima templado. El calentamiento global provocará una disminución de la superficie con acumulación de 600 a 800 HF de 11.7, 25.8 y 33\% en los escenarios climáticos 2030, 2050 y 2070 del RCP 4.5, respectivamente y hasta $99.5 \%$ la superficie con acumulación de 800 a $900 \mathrm{HF}$ en el clima 2070 del RCP 8.5. En cambio, la superficie con poca acumulación de HF (50 a 200) aumentará 11.5, 29.3 y 36.4\% en los escenarios climáticos 2030, 2050 y 2070 del RCP 4.5, respectivamente. Los frutales caducifolios en el futuro se verán limitado por la disminución de HF, en las áreas con mayor acumulación de frío, de igual manera pueden ser afectados los cereales de otoño-invierno.

Palabras clave: calentamiento global, frutales caducifolios, tendencia histórica, RCP.

Recibido: junio de 2019

Aceptado: agosto de 2019 


\section{Introducción}

En México los frutales caducifolios se cultivan en el centro y norte del país donde la temperatura, al final del otoño y durante el invierno, es lo suficientemente baja para que ocurra una defoliación de los árboles y presenten un letargo durante el invierno. Una vez que los árboles alcanzan el letargo, empieza la acumulación de frío durante el invierno, el cual es indispensable para la floración en la primavera e inicio de un nuevo ciclo vegetativo y reproductivo. Esta particularidad de los frutales caducifolios ha forzado a buscar nichos ecológicos que permitan el desarrollo adecuado de cultivos como el chabacano, ciruelo, durazno, manzano y vid, entre otros (Martínez, 2012; Zegbe et al., 2016).

El calentamiento global tiene el potencial de reducir el frío disponible en el invierno y afectar la producción de los árboles frutales caducifolios. Diversos investigadores han pronosticado la disminución en la acumulación de horas frío en diversas regiones del mundo, en escenarios climáticos futuros, utilizando modelos de circulación general u otras metodologías (Jindal y Mankotia, 2004; Baldocchi y Wong 2008; Luedeling et al., 2009a; Yu et al., 2010; Luedeling et al., 2011; Medina-García et al., 2011).

Actualmente el cambio climático representa uno de los principales problemas que enfrenta la agricultura, debido a las alteraciones que causa en los patrones del clima (IPCC, 2014) debido al incremento en la concentración atmosférica de los gases efecto invernadero (GEI). Con base a, escenarios que no asumen las políticas climáticas para reducir las emisiones de gases de efecto invernadero, se prevé que la temperatura media mundial aumente entre 2 y $5{ }^{\circ} \mathrm{C}$ para finales de este siglo (IPCC, 2014), esto afecta directamente la agroclimatología relacionada con el manejo de las actividades agrícolas (Ruiz-Corral et al., 2016).

La temperatura de las áreas agrícolas de México se ha venido incrementando de manera perceptible desde la década de los años noventa del siglo pasado (Ruiz et al., 2010; Zarazúa-Villaseñor et al., 2011a), este incremento de la temperatura trae consigo modificaciones en variables agroclimáticas (Zarazúa-Villaseñor et al., 2011b) como puede ser la acumulación de frío en el periodo invernal (Medina-García et al., 2011; Santillán-Espinoza et al., 2011).

La acción que ejercen las bajas temperaturas no siempre es perjudicial, debido a que las especies caducifolias requieren un período de enfriamiento necesario para el óptimo desarrollo tanto de yemas vegetativas como reproductivas (Warmund y Krumme, 2005; Luedeling et al., 2009a). Sin embargo, en climas subtropicales la falta de frío en el invierno causa una brotación errática e irregular, con la consiguiente reducción en la cantidad y calidad de la fruta (Ramírez et al., 2011; Medina-García et al., 2014), lo cual indica que el requerimiento de frío en especies frutales caducifolias es un factor decisivo en la adaptación de estas especies a su ambiente.

El método convencional (Weinberger, 1950) es el método de cuantificación de frío más utilizado, contempla la suma diaria de horas en que la temperatura está entre 0 y $7.2{ }^{\circ} \mathrm{C}$ durante el otoño e invierno (Pérez et al., 2008; Okie y Blackburn, 2011). Con este método se han establecido los requerimientos de frío de diversos frutales, relacionando la acumulación de horas frío con la brotación regular del árbol. Este es un valor estándar que aún se utiliza. 
Con el fin de lograr una adecuada planeación del desarrollo agrícola a corto y mediano plazo en una región, es necesario estimar en escenarios climáticos futuros, la disponibilidad de recursos agroclimáticos, entre ellos la acumulación de frío, para contar con suficiente información y poder diseñar e implementar medidas de adaptación que eviten o minimicen en lo posible los impactos negativos del cambio climático (Medina-García et al., 2014).

\section{Materiales y métodos}

Se utilizaron datos de las estaciones de clima del Servicio Meteorológico Nacional de la República Mexicana. Se seleccionaron 2854 estaciones con más de 20 años y más de $90 \%$ de datos en el periodo de 1961 a 2010 distribuidas en todo el país. Con los datos diarios de temperatura máxima y mínima, se obtuvieron datos horarios (Snyder, 1985) de toda la serie histórica de datos de las estaciones. Con estos datos se estimó el número de horas en que la temperatura estuvo entre 0 y $7.2^{\circ} \mathrm{C}$, en cada día, que corresponden a las horas frío (HF) de acuerdo a Weinberger (1950). Se sumó el número de HF para cada uno de los meses de la temporada invernal (noviembre a febrero) para cada uno de los años de la serie de cada estación.

Para el análisis de tendencias históricas de HF se seleccionaron únicamente estaciones con acumulación de al menos $50 \mathrm{HF}$ en todo el periodo invernal en promedio en toda la serie, resultando 1688 estaciones que cumplieron con este requisito (Figura 1). Se hicieron análisis de tendencias históricas para cada mes y para el periodo invernal completo utilizando promedios móviles de 20 años. Para corroborar las tendencias de los promedios móviles se utilizó la prueba no paramétrica de Mann-Kendal (Mann, 1945; Kendall, 1975), ya que se considera la más adecuada para el análisis de tendencias en series temporales climatológicas (Miró et al., 2009; Ahmed et al., 2014; de Melo et al., 2015) y se complementó con la estimación Sen de pendiente de la tendencia (Sen, 1968) para esto se utilizó la macro Makesens, desarrollada en el Finish Meteorologial Institute (Salmi et al., 2002).

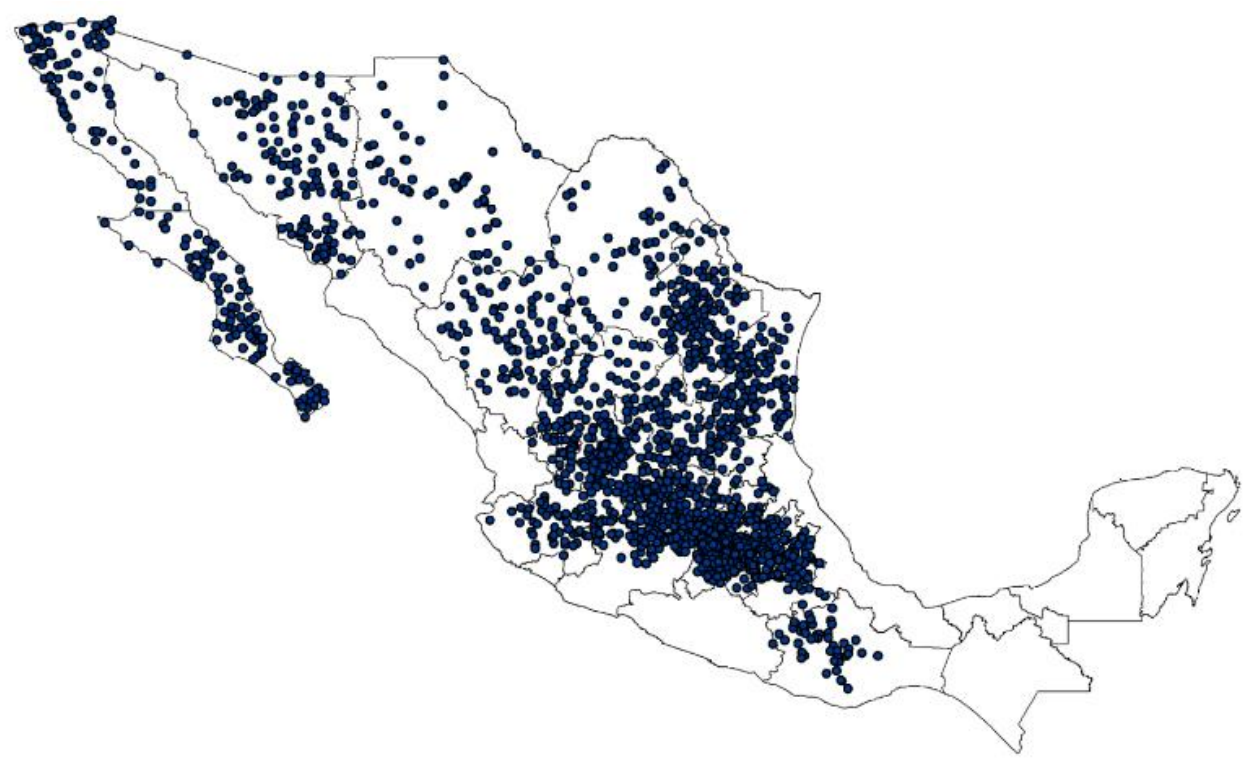

Figura 1. Estaciones climáticas utilizadas en el análisis de tendencias históricas de HF. 
Para generar mapas con el número de HF a partir de la temperatura mínima media mensual, se obtuvieron modelos para cada mes tomando como variable independiente la temperatura mínima media y como variable dependiente las HF acumuladas en el mes correspondiente, utilizando las 2 854 estaciones seleccionadas inicialmente. Uno de los patrones de crecimiento más observados en las poblaciones naturales es el crecimiento logístico y se representa con una curva sigmoidea.

En este modelo hay una fase en que el crecimiento de la población es relativamente lento, seguida de una fase de aceleración, luego, la tasa de crecimiento se hace más lenta y finalmente se estabiliza (Ibarra y Rodríguez, 2010). Al graficar los datos de HF se observó un comportamiento logístico, por lo que se probaron diferentes modelos sigmoides para ajustar los datos.

Para estimar el efecto del cambio climático en el número de HF se integró un modelo ensamble a partir del valor de la mediana de 11 modelos de circulación general (MCG) reducidos en escala y calibrados (Walton et al., 2013) y pertenecientes al CMIP5 (Intercomparación de modelos acoplados fase 5) reportados en la $5^{\mathrm{a}}$ entrega del IPCC: (BCC-CSM1-1, CCSM4, GISS-E2-R, HadGEM2-AO, HadGEM2-ES, IPSL-CM5A-LR, MIROC-ESM-CHEM, MIROC-ESM, MIROC5, MRI-CGCM3, NorESM1-M), los cuales fueron obtenidos a partir de información del portal de datos de Cambio Global de WorldClim.

El ensamble se generó bajo dos rutas de concentración (RCP) de gases efecto invernadero esto es, se utilizó un RCP de emisiones intermedias (4.5) el cual es consistente con un futuro con reducción de emisiones relativamente ambiciosas y un RCP de emisiones altas (8.5), que es coherente con un futuro sin cambios de política para reducir las emisiones (Van Vuuren et al., 2011).

Se utilizaron los valores mensuales del ensamble de los 11 modelos de temperatura mínima media mensual de los años 2021 a 2080, para los escenarios 2021-2040, 2041-2060 y 2061-2080, en adelante referidos como climas o años 2030, 2050 y 2070 respectivamente. Se tomó como clima base o de referencia la misma variable en el periodo 1961-2010 del sistema de información climática del INIFAP (Ruiz-Corral et al., 2016). Se generaron imágenes temáticas raster con una resolución de 30" arco, correspondientes a valores mensuales de temperatura mínima del clima base y de los escenarios de noviembre a febrero. Finalmente, de acuerdo con los resultados obtenidos, se proponen algunas medidas de adaptación a los escenarios climáticos estudiados.

\section{Resultados y discusión}

\section{Tendencias históricas}

Para validar la estimación de los datos horarios de temperatura se ajustaron a un modelo de regresión lineal con datos horarios reales, resultando una $\mathrm{R}^{2}=0.9$, cumpliendo con los supuestos de validación 1:1; es decir, el intercepto fue estadísticamente igual a cero y la pendiente estadísticamente igual a uno (Fritz et al., 1997).

Con las HF de cada uno de los meses de la temporada invernal se hicieron análisis de tendencias históricas de 1961 a 2010 con promedios móviles cada 20 años, considerando 1688 estaciones (Figura 2). El número de HF en noviembre presenta una tendencia a la disminución $(p \leq 0.0001)$; es decir, el número promedio de HF de este mes en México ha disminuido $11.8 \mathrm{~h}$ en los 50 años de estudio, $0.24 \mathrm{~h}$ por año (Figure $2 \mathrm{~A}$ ). 
La tendencia histórica del número promedio de $\mathrm{HF}$ en el mes de diciembre es en promedio ligeramente hacia el aumento; es decir, ha aumentado 1 el número de $\mathrm{HF}$ en los 50 años de estudio, $0.02 \mathrm{~h}$ por año; sin embargo, el modelo no resultó significativo ( $p=0.3718$ ) (Figura 2B). Este comportamiento se debe a que en el mes de diciembre se presentó, de manera general, menor acumulación de HF en el periodo de 1976 a 1995, para luego regresar a la acumulación normal o ligeramente mayor de HF (Figura 2B). En la Figura 2C se presenta la tendencia de HF en enero, la cual es hacia la disminución ( $p \leq 0.0001)$, lo que ha ocasionado una pérdida de $15.2 \mathrm{HF}$ en 50 años.

A)

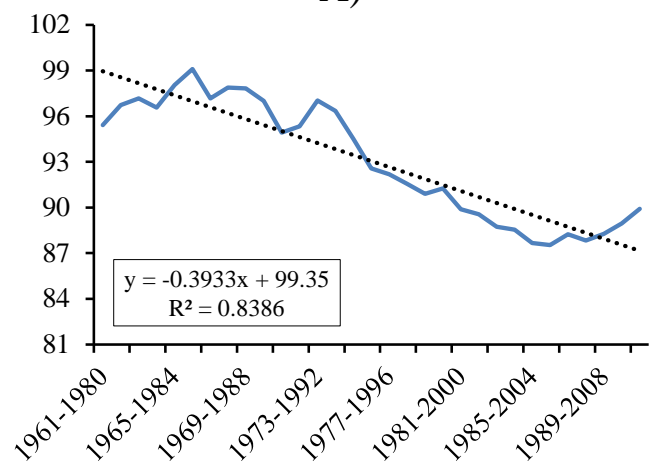

C)

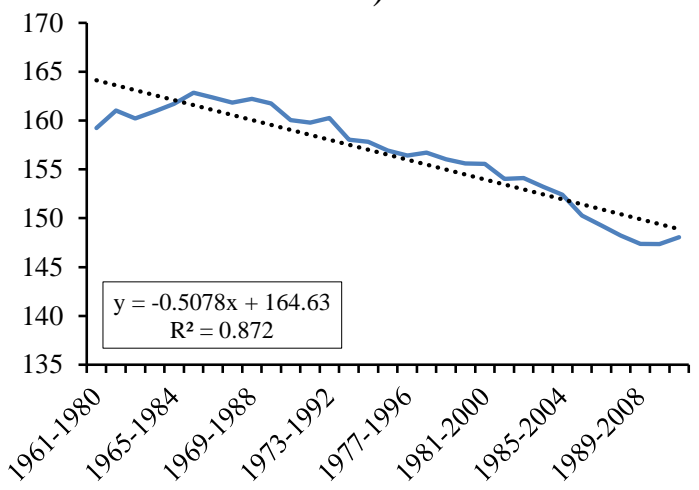

B)

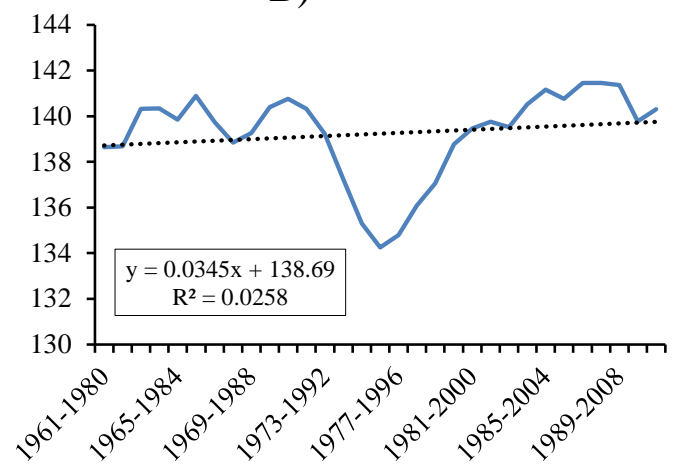

D)

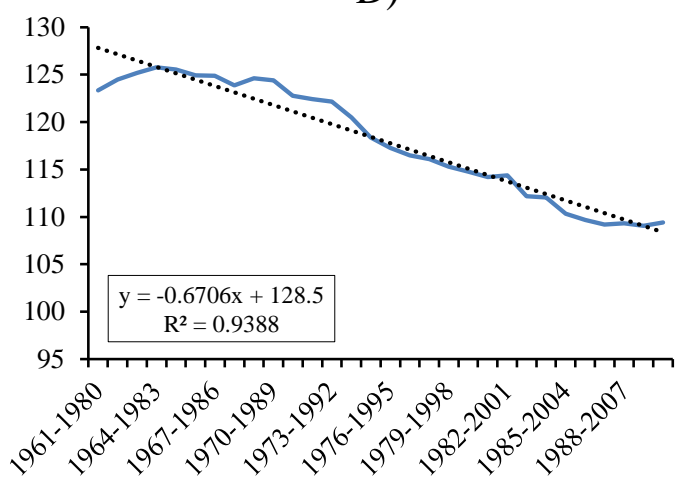

E)

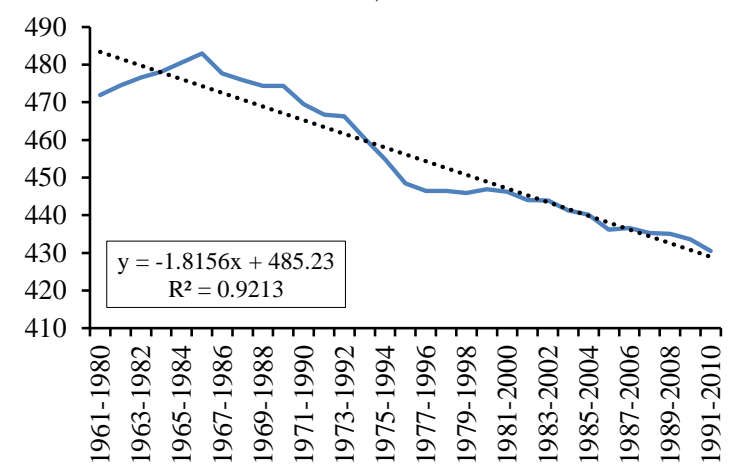

Figura 2. Tendencia histórica del número de $\mathrm{HF}\left(0\right.$ a $\left.7.2^{\circ} \mathrm{C}\right)$. A) noviembre; $\left.\mathrm{B}\right)$ diciembre; C) enero; D) febrero; y E) para las HF acumuladas de noviembre a febrero en México. Las unidades del eje horizontal son años (periodos de 20 años) y las unidades del eje vertical son número de HF. 
La tendencia histórica del número promedio de HF en el mes de febrero es también hacia la baja ( $p \leq 0.0001$ ), con una disminución promedio de $20.1 \mathrm{HF}$ en 50 años (Figura 2D). Finalmente, en la Figura $2 \mathrm{E}$ se presenta la tendencia del número promedio de $\mathrm{HF}$ en el periodo invernal de noviembre a febrero ( $p \leq 0.0001)$ y es hacia la baja, con una disminución promedio de $45.3 \mathrm{HF}$ en el periodo de 1961 a 2010. Claramente se observa un efecto del cambio climático al presentar el análisis de tendencias históricas en tres de los cuatro meses y el periodo invernal acumulado una disminución del número de $\mathrm{HF}$, lo cual tiene un efecto desfavorable en la producción de los frutales de clima templado (Medina-García et al., 2011; Ramírez et al., 2011).

El análisis de tendencias históricas con la prueba de Mann-Kendall reflejó resultados similares a los promedios móviles. En los cuatro meses y el acumulado, las tendencias resultaron iguales, noviembre, enero, febrero y noviembre-febrero con tendencia negativa y diciembre con tendencia positiva, aunque en todos los caos con valores de la pendiente ligeramente inferiores. La significancia resultó igual, excepto en el mes de noviembre donde resultó no significativo con esta prueba (Cuadro 1).

Cuadro 1. Prueba de tendencia de Mann-Kendall y estimación de pendiente Sen para las HF por mes y acumuladas en la temporada de invierno noviembre-febrero.

\begin{tabular}{cccccc}
\hline \multicolumn{2}{c}{ Tendencia Mann-Kendall } & & \multicolumn{3}{c}{ Pendiente estimada Sen } \\
\cline { 1 - 2 } \cline { 5 - 6 } Serie & Estadístico Z & & Significancia & Pendiente (Q) & Constante (B) \\
\hline Enero & -2.23 & & $*$ & -0.279 & 162.558 \\
Febrero & -3.26 & & $* *$ & -0.422 & 128.366 \\
Noviembre & -0.45 & & $\mathrm{~ns}$ & -0.064 & 96.002 \\
Diciembre & 1.24 & & $\mathrm{~ns}$ & 0.177 & 136.582 \\
Noviembre-Febrero & -1.76 & & $*$ & -0.647 & 525.151 \\
\hline
\end{tabular}

Nivel de significancia 90\% (*); 95\% (**); 99\% (***); ns (no significativo).

Luedeling et al. (2009b) encontraron en la Península Arábiga disminuciones en la acumulación de frío $\left(0\right.$ a $7.2^{\circ} \mathrm{C}$ ), en el orden de 1.02 a 9.05 h año entre 1983 y 2008, en México se encontró de igual manera disminución de frío, pero en el orden de 0.91 HF/año en el periodo 1961-2010, el cual corresponde con el límite inferior de estos autores. Con esta tendencia de pérdida de 9 $\mathrm{HF} /$ década, en un futuro cercano la producción de las especies que requieren frío podría llegar a ser marginal o imposible, tal como lo señalan Grageda et al. (2016) para nogal.

\section{Modelos de estimación de HF}

Para el análisis de escenarios climáticos futuros, es necesario generar mapas con el número promedio de HF de cada mes, para esto se obtuvieron modelos mensuales para estimar las HF a partir de la temperatura mínima promedio utilizando las 2854 estaciones seleccionadas inicialmente. Los modelos que mejor se ajustaron a la dispersión de los datos (Figura 3), fueron los modelos logísticos de tres parámetros. Los cuatro modelos presentaron valores de $\mathrm{R}^{2}=0.95$ lo cual indica muy buen ajuste. En el Cuadro 2 se presentan los modelos para los cuatro meses. 


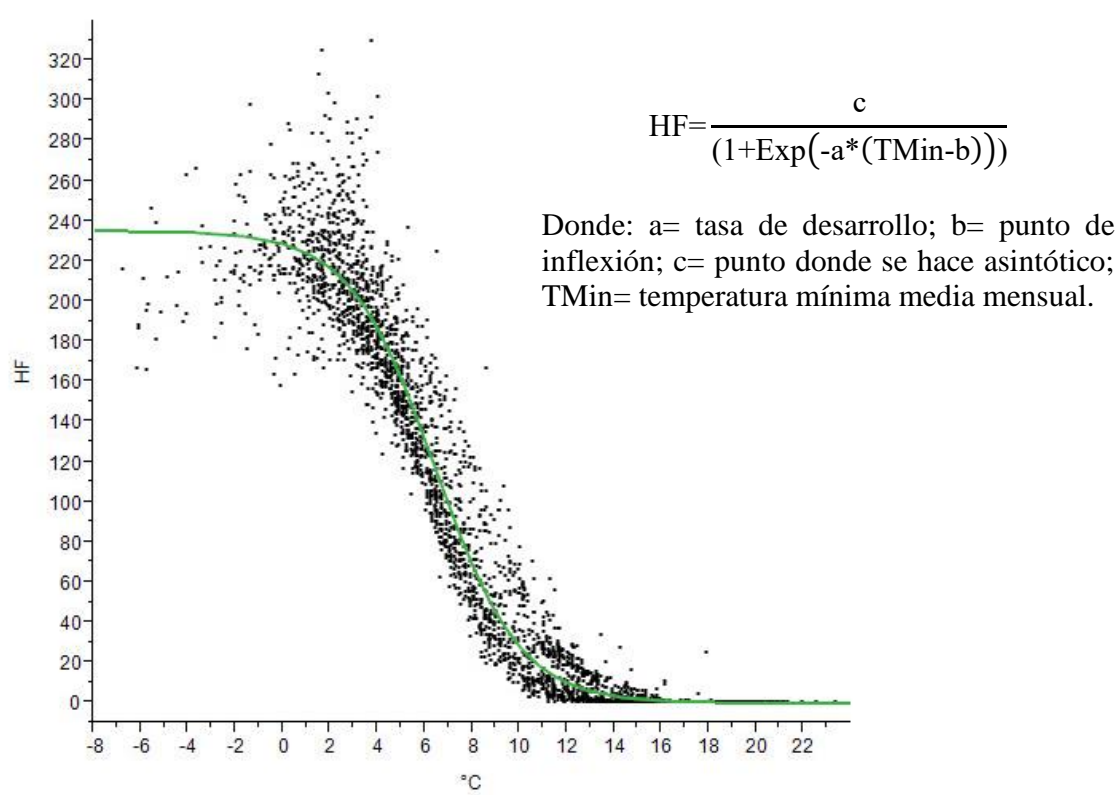

Figura 3. Gráfica de dispersión de datos de HF de enero y modelo logístico de tres parámetros.

Cuadro 2. Modelos logísticos de tres parámetros para estimar las HF de cada uno de los meses del periodo invernal a partir de la temperatura mínima media.

\begin{tabular}{ccccc}
\hline Mes & Tasa de crecimiento & Punto de inflexión & Asíntota & $\mathrm{R}^{2}$ \\
\hline Noviembre & -0.505918 & 6.190316 & 220.81403 & 0.959637 \\
Diciembre & -0.539329 & 6.386607 & 231.75049 & 0.952652 \\
Enero & -0.55736 & 6.406391 & 235.2206 & 0.948577 \\
Febrero & -0.532459 & 6.147172 & 211.53101 & 0.955979 \\
\hline
\end{tabular}

\section{Escenarios climáticos}

Con los modelos obtenidos y utilizando el sistema de información geográfica Idrisi Selva (Eastman, 2012), se generaron imágenes nacionales de HF para cuatro meses de la temporada invernal para el clima base (1961-2010) y para los climas 2030, 2050 y 2070 en cada uno de los RCP 4.5 y 8.5.

En la Figura 4A se muestran los mapas con el número de HF promedio en el periodo invernal (noviembre a febrero) en la República Mexicana. Actualmente los frutales de clima templado se cultivan principalmente en las zonas que tienen entre 600 a $900 \mathrm{HF}$, de acuerdo al mapa de la Figura 4A, el manzano se cultiva en zonas de 800 a $900 \mathrm{HF}$, otros como durazno, ciruelo y chabacano se cultivan también en ese mismo rango de HF, pero también en las zonas de 600 a $800 \mathrm{HF}$.

En el escenario del clima 2030 del RCP 4.5 (Figura 4B) la superficie de los rangos sin frío, 50-200, $200-400$ y 400 a $600 \mathrm{HF}$ aumenta 10, 11.5, 5.1 y 12.1\%, respectivamente (Cuadro 3) con respecto al escenario actual; es decir, la superficie con menor acumulación de frío, donde no se cultivan frutales de clima templado, es probable que aumente, mientras que la superficie con mayor acumulación de frío, donde se cultivan frutales de clima templado, presenta una disminución en el rango $600-800 \mathrm{HF}$ de $11.7 \%$ y en el rango de $800-900 \mathrm{HF}$ una disminución de $30.5 \%$. 
En el clima 2050 del RCP 4.5 (Figura 4C) la superficie de los rangos sin frío, 50-200, 200-400 y 400 a 600 HF y la superficie con mayor acumulación de frío (600-800 y 800-900 HF), presentan una tendencia similar al clima 2030 pero más acentuado, así se tiene que la superficie del rango $50-200 \mathrm{HF}$ es probable que aumente $29.3 \%$ y la superficie del rango $800-900 \mathrm{HF}$ es probable que disminuya $57.1 \%$ (Figura 4C y Cuadro 3).

A) Escenario actual

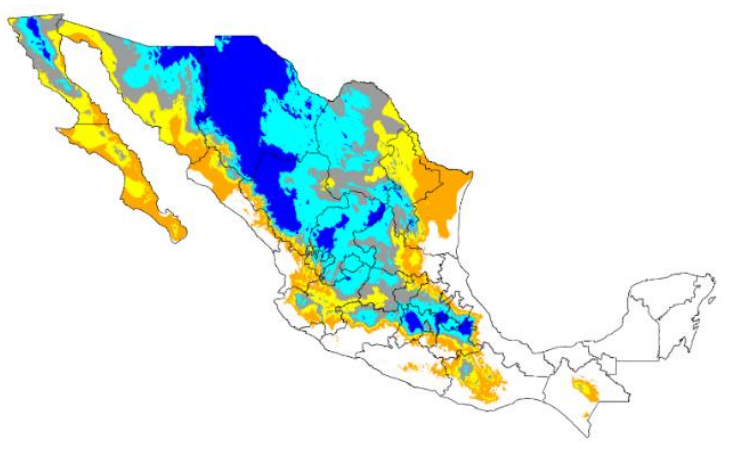

C) Escenario 2050

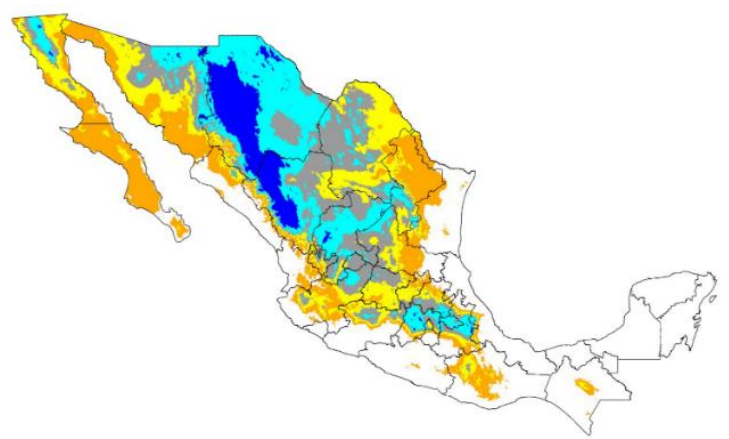

B) Escenario 2030

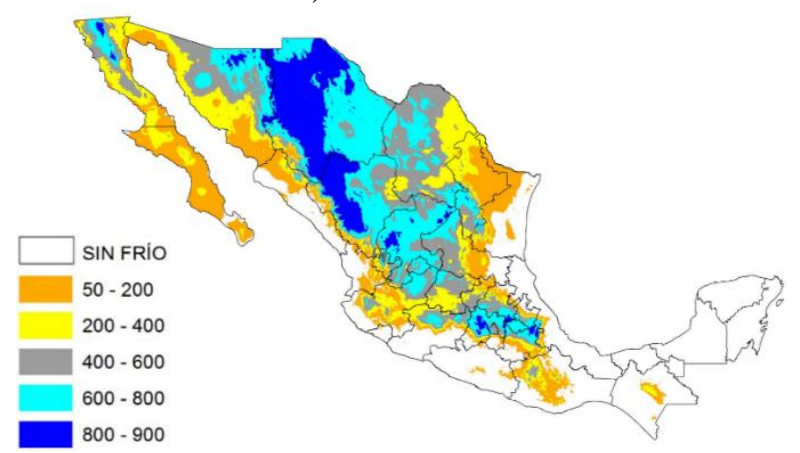

D) Escenario 2070

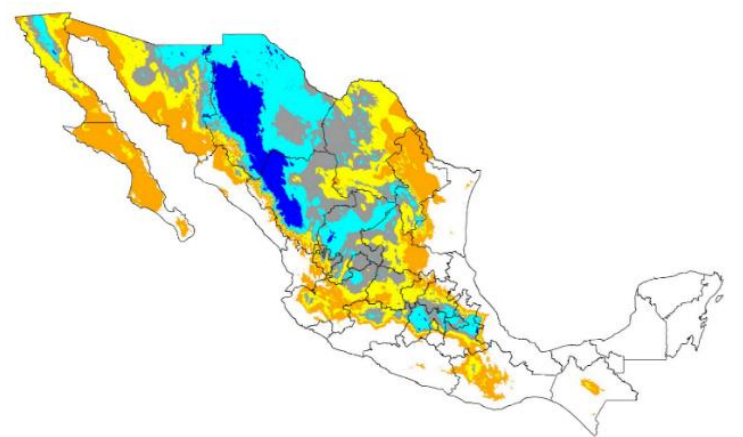

Figura 4. Número de HF promedio en el periodo invernal (noviembre a febrero) en el escenario actual y en tres escenarios climáticos futuros del RCP 4.5, en la República Mexicana.

En la Figura 4D se presenta el mapa de las HF en el clima 2070 del RCP 4.5, al igual que en los climas anteriormente descritos el porcentaje de la superficie con menor acumulación de frío aumentará hasta $36.4 \%$ en el rango 50-200 HF mientras que, el porcentaje de superficie con mayor acumulación de frío disminuirá hasta 61.6\% en el rango 800-900 HF (Figura 4D y Cuadro 3).

Cuadro 3. Porcentaje de cambio de la superficie con HF en la temporada invernal (noviembre a febrero) en escenarios climáticos futuros con respecto al escenario actual en la República Mexicana.

\begin{tabular}{lccccccc}
\hline & \multicolumn{3}{c}{ RCP 4.5} & & \multicolumn{3}{c}{ RCP 8.5} \\
\cline { 2 - 4 } \cline { 6 - 8 } & 2030 & 2050 & 2070 & & 2030 & 2050 & 2070 \\
\hline Sin frío & 10 & 18.7 & 23.9 & & 18.8 & 40.2 & 56.1 \\
$50-200$ & 11.5 & 29.3 & 36.4 & & 27.1 & 43.5 & 59.8 \\
$200-400$ & 5.1 & 14.9 & 14.7 & & 16.2 & 42.6 & 60 \\
$400-600$ & 12.1 & 16.1 & 14.1 & & 29.2 & 34.3 & -9.6 \\
$600-800$ & -11.7 & -25.8 & -33 & & -26.4 & -67.9 & -78.3 \\
$800-900$ & -30.5 & -57.1 & -61.6 & & -68.6 & -94 & -99.5 \\
\hline
\end{tabular}


Las áreas de alta acumulación de frío (600-800 y 800-900 HF) tendrán una fuerte reducción conforme se avance en los escenarios hacia el futuro, especialmente en áreas de la sierra de Baja California, sierra Madre Occidental sus estados adyacentes como son Sonora, Chihuahua, Coahuila, Durango, Zacatecas y Aguascalientes y áreas del eje Neo Volcánico, por lo que es posible que en esas regiones se inicie una reducción de la aptitud de la superficie agrícola para frutales de clima templado tal como lo señalan Medina et al. (2011) en la región manzanera de Chihuahua.

La reducción de las HF se debe al aumento de la temperatura lo que coincide con lo señalado por Cavazos et al. (2012), en el sentido de que será conveniente el uso de variedades de frutales de clima templado con menor requerimiento de HF.

En la Figura 5A se muestran los mapas con el número de HF promedio en el periodo invernal (noviembre a febrero) en la República Mexicana. En el clima 2030 del RCP 8.5 (Figura 5B) la superficie de los rangos SIN FRÍO, 50-200, 200-400 y 400 a 600 HF aumenta 18.8, 27.1, 16.2 y 29.2\% (Cuadro 3), respectivamente, con respecto al escenario actual; es decir, la superficie con menor acumulación de frío, donde no se cultivan frutales de clima templado, es probable que aumente, mientras que la superficie con mayor acumulación de frío, donde se cultiva la mayor parte de frutales de clima templado, presenta una disminución en el rango $600-800 \mathrm{HF}$ de $26.4 \%$ y en el rango de 800-900 HF una disminución de 68.6\%.

En el clima 2050 de la RCP 8.5 (Figura 5C) la superficie de los rangos SIN FRÍO, 50-200, 200400 y 400 a $600 \mathrm{HF}$ y la superficie con mayor acumulación de frío (600-800 y 800-900 HF), presentan una tendencia similar al clima 2030 pero más acentuado, así se tiene que la superficie del rango 50-200 HF es probable que aumente $40.2 \%$ y la superficie del rango $800-900 \mathrm{HF}$ es probable que disminuya hasta $94 \%$ (Figura 4C y Cuadro 3 ).

En la Figura 5D se presenta el mapa de HF en el clima 2070 de la RCP 8.5, al igual que en los climas anteriormente descritos el porcentaje de la superficie con menor acumulación de frío aumentará hasta $60 \%$ en el rango 200-400 HF, mientras que, el porcentaje de superficie con mayor acumulación de frío disminuirá hasta 99.5\% en el rango 800-900 HF, prácticamente desaparecerá (Figura 5D y Cuadro 3). En este último escenario, solo en la Sierra Madre Occidental y en algunas pequeñas áreas del eje neo volcánico es probable que se acumulen entre 600 y $800 \mathrm{HF}$.

En general en las RCP 4.5 y 8.5 se presenta el mismo comportamiento de reducción y aumento de superficie en los diferentes rangos de HF, pero en la RCP 8.5 los aumentos o disminuciones son más drásticos, esto debido a que este es un escenario de emisiones altas de GEI; es decir, que en este escenario no hay cambios de política para reducir las emisiones (Van Vuuren et al., 2011), por lo que con mayores emisiones de GEI habrá mayor efecto invernadero y mayor aumento de temperatura de acuerdo al modelo de la RCP.8.5.

Estos resultados concuerdan con Baldochi y Wong (2008) en California donde para el año 2100 esperan 500 horas menos de frío en el periodo invernal. De igual manera Luedeling et al. (2009a) encontraron resultados similares para California y para la Península Arábiga (Luedeling et al., 2009 b). Este autor también probó diferentes modelos de estimación de frío en California y en todos los casos encontró disminución de frío invernal (Luedeling et al., 2009c). Resultados similares se encontraron en México en la zona manzanera de Chihuahua (Medina-García et al., 2011; Ramírez et al., 2011) y en durazno (Ramírez-Legarreta et al., 2008; Medina-García et al., 2014). 
A) Escenerio actual

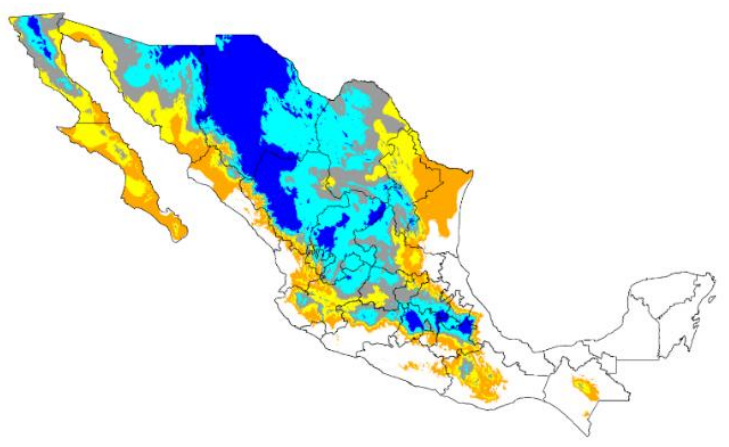

C) Escenario 2050

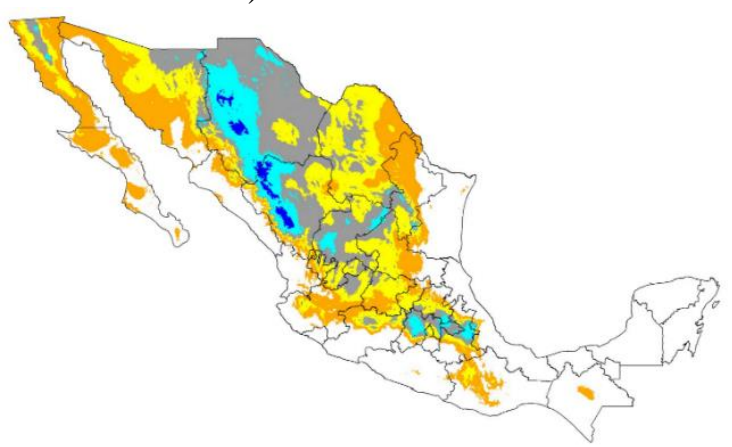

B) Escenerio 2030

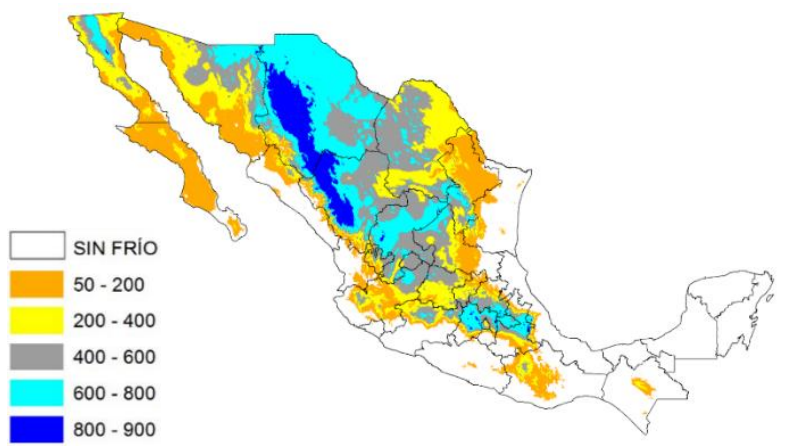

D) Escenario 2070

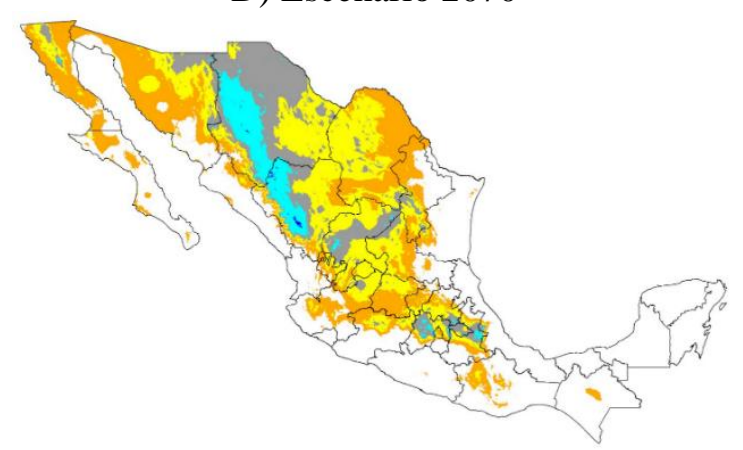

Figura 5. Número de HF promedio en el periodo invernal (noviembre a febrero) en el escenario actual y en tres escenarios climáticos futuros del RCP 8.5, en la República Mexicana.

Si la reducción de HF como se observa en todos los escenarios es a consecuencia del aumento de temperatura, se observarán en el futuro probables afectaciones a los cultivos de otoño-invierno, no solo frutales de clima templado, que incluso podrían desaparecer de este ciclo en algunas regiones, lo que afectaría especialmente a aquellas especies que requieren de cierto tiempo de vernalización, tal como es el caso del trigo, que es uno de los principales cultivos en este ciclo. Se sabe que las HF alargan los períodos fenológicos al reducir la velocidad con que se llevan a cabo los procesos fisiológicos y consecuentemente retardan el crecimiento. El trigo de invierno experimentará un acortamiento de las etapas siembra-floración y floración-madurez fisiológica, como respuesta a la rápida acumulación de unidades calor (Félix et al., 2009; Zarazúa-Villaseñor et al., 2011a).

De acuerdo a lo anterior, es necesario iniciar la investigación en mejoramiento genético de cultivos anuales y frutales caducifolios para adaptarse a condiciones futuras, debido que el cambio climático no es un fenómeno próximo a suceder, sino que ya está ocurriendo, tal como lo demuestran estudios de análisis retrospectivo del clima en áreas agrícolas del país realizados por Ruiz et al. (2000); Zarazúa-Villaseñor et al. (2011a); Medina-García et al. (2011), Medina-García et al. (2017).

\section{Conclusiones}

Se observó un efecto desfavorable del calentamiento global en el periodo histórico 1961-2010 al disminuir en promedio para todo el país 11.8 HF en el mes de noviembre, 15.2 HF en el mes de enero y $20.1 \mathrm{HF}$ en el mes de febrero, solo en el mes de diciembre aumentó en promedio $1 \mathrm{HF}$. Considerando todo el periodo invernal de noviembre a febrero ha ocurrido una disminución promedio de $45.3 \mathrm{HF}$ en los 50 años de estudio. 
Las áreas de alta acumulación de frío (600-800 y 800-900 HF) tendrán una fuerte reducción conforme se avance en los escenarios hacia el futuro, especialmente en áreas de la sierra de Baja California, sierra Madre Occidental y áreas del eje Neo Volcánico, por lo que es posible que en esas regiones se inicie una reducción de la aptitud de la superficie agrícola para frutales de clima templado y algunos cultivos del ciclo otoño-invierno.

Considerando los efectos del calentamiento global en los escenarios climáticos futuros en los RCP 4.5 y 8.5, la superficie con mayor acumulación de HF va en disminución hacia el futuro, lo que repercutirá en la falta de frío en el invierno causando una brotación errática e irregular, con la consiguiente reducción en la cantidad y calidad de la fruta, por lo que los requerimientos de frío en especies frutales caducifolias deben tomarse en cuenta en la adaptación al cambio climático.

Los resultados de este estudio pueden coadyuvar de base en el diseño de estrategias, para enfrentar el cambio climático en las áreas productoras de frutales de clima templado en México, como pudiera ser la generación de nuevas variedades con menor requerimiento de HF.

\section{Literatura citada}

Ahmed, S. I.; Rudra, R.; Dickinson, T. and Ahmed, M. 2014. Trend and periodicity of temperature time series in Ontario. Am. J. Climate Change. 3:272-288.

Baldocchi, D. and Wong, S. 2008. Accumulated winter chill is decreasing in the fruit growing regions of California. Climatic Change. 87(1):153-166.

Cavazos, P. M. T.; Lizárraga, P. M. L.; Hernández, M. R.; Kretschmar, T.; Pavía, E. G.; Valenzuela, E.; Rodríguez, M. V. M.; Espinoza, T. B. E.; Rete, C. A. y Figueroa, Y. G. 2012. Situación actual y bajo escenarios de cambio climático de la industria vitivinícola de Baja California, México. Reporte Final del Proyecto. Instituto Nacional de Ecología (INE) y CICESE. Ensenada, BC. 75 p.

Eastman, J. R. 2012. IDRISI selva manual. Clark labs - Clark University. Worcester, Mass. USA. $322 \mathrm{p}$.

Félix, V. P.; Ortíz, E. J. E.; Fuentes D. G.; Quintana, Q. J. G. y Grageda, G. J. 2009. Horas frío en relación al rendimiento de trigo: Áreas de producción del estado de Sonora. Folleto técnico núm. 63. INIFAP-CIRNO-CEVY. Cd. Obregón, Sonora, México. 40 p.

Fritz, J. O.; Vanderlip, R. L.; Heiniger, R. W. and Abelhalim, A. Z. 1997. Simulating forage sorghum yields with SORKAM. Agron. J. 89:64-68.

Grageda, G. J.; Ruiz, C. J. A.; García, R. G. E; Núñez, M. J. H.; Valenzuela, L. J.; Ruiz Á. O. y Jiménez, L. A. 2016. Efecto del cambio climático en la acumulación de horas frío en la región nogalera de Hermosillo, Sonora. Rev. Mex. Cienc. Agríc. 13(Pub. Esp.):2487-2495.

Ibarra, U. J. T. y Rodríguez, C. J. 2010. El modelo logístico: una alternativa para el estudio del crecimiento poblacional de organismos. Rev. Elec. Veterinaria. 11(3):1695-7504.

IPCC. 2014. Intergovernmental Panel on Climate Change. Climate Change 2014: synthesis report. Contribution of working groups $\mathrm{i}$, ii and iii to the fifth assessment report of the intergovernmental panel on climate change [core writing team. Pachauri, K. R. and Meyer, L. A. (Eds.)]. IPCC, Geneva, Switzerland. 151 p.

Jindal, K. K. and Mankotia, M. S. 2004. Impact of changing climatic conditions on chilling units, physiological attributes and productivity of apple in western Himalayas. Acta Hortic. 662:111-117. 
Kendall, M. G. 1975. Rank correlation methods. $4{ }^{\text {th }}$ Edition, Charles Griffin, London. 202 p.

Luedeling, E.; Gebauer, J. and Buerkert, A. 2009b. Climate change effects on winter chill for tree crops with chilling requirements on the Arabian Peninsula. Climatic Change. 96(12):219-237.

Luedeling, E.; Girvetz, E. H.; Semenov, M. A. and Brown, P. H. 2011. Climate change affects winter chill for temperate fruit and nut trees. PLoS ONE. 6(5):20155-1371.

Luedeling, E.; Zhang, M. and Girvetz, E. H. 2009a. Climatic changes lead to declining winter chill for fruit and nut trees in California during. PLoS ONE 4(7):1950-2099. e6166.

Luedeling, E.; Zhang, M.; Luedeling, V. and Girvetz, E. H. 2009c. Sensitivity of winter chill models for fruit and nut trees to climatic changes expected in California's Central Valley. Agric. Ecosys. Environ. 133(1):23-31.

Mann, H. B. 1945. Non-parametric test against trend. Econometrica. 13(3):245-259.

Martínez, D. G. 2012. La brotación de la vid en Sonora. INIFAP-CIRNO-CECH. Hermosillo, Sonora. Folleto técnico núm. 44. 26-27 pp.

Medina, G. G.; Mena, C. J.; Ruiz, C. J. A.; Rodríguez, M. V. M. y Soria, R. J. 2017. El cambio climático afecta el número de horas del rango térmico del chile (Capsicum annuum L.) en el Norte-Centro de México. Rev. Mex. Cienc. Agríc. 8(8):1797-1812.

Medina, G. G.; Ruiz, C. J. A.; Ramírez, L. M. R. y Díaz, P. G. 2011. Efecto del cambio climático en la acumulación de frío en la región manzanera de Chihuahua. Rev. Mex. Cienc. Agríc. 2(Pub. Esp.):195-207.

Medina, G. G.; Ruiz, C. J. A.; Zegbe, D. J. A.; Soria, R. J.; Rodríguez, M. V. y Díaz, P. G. 2014. Impacto potencial del cambio climático en la región productora de durazno en Zacatecas, México. Rev. Mex. Cienc. Agríc. 10(Pub. Esp.):1939-1950.

Melo, T. M.; Louzada, J. A. S. and Pedrollo, O. C.; 2015. Trends in Extreme Indices and Seasonal Analysis of Precipitation and Temperature in the Northwest Region of Rio Grande do Sul, Brazil. American Journal of Climate Change. 4:187-202.

Miró, J.; Estrela, M. J.; Pastor, F. y Millán, M. 2009. Análisis comparativo de tendencias en la precipitación, por distintos inputs, entre los dominios hidrológicos de la Segura y del Júcar. Investigaciones Geográficas. 49:129-157.

Okie, W. R. and Blackburn, B. 2011. Increasing chilling reduces heat requirement for floral budbreak in peach. Hortscience. 46(2):245-252.

Pérez, F. J.; Ormeño, N. J.; Reynaert, B. and Rubio, S. 2008.Use of the dynamic model for the assessment of winter chilling in a temperate and a subtropical climatic zone of Chile. Chilean J. Agric. Res. 68(2):198-206.

Ramírez, L. M. R.; Jacobo, C. J. L.; Gardea, B. A. A. y Parra, Q. R. A. 2008. Modelo de desarrollo floral en manzanos [Malussylvestris (L.) Mill. var. Domestica (Borkh) Mansf.] Red Delicious y Golden Delicious como herramienta de toma de decisiones en el manejo integrado de enfermedades. Rev. Mex. Fitopatol. 26(2):153-163.

Ramírez, L. M. R.; Ruiz, C. J. A.; Medina, G. G.; Jacobo, C. J. L.; Parra, Q. R. A.; Ávila, M. M. R. y Amado, A. J. P. 2011. Perspectivas del sistema de producción de manzano en Chihuahua, ante el cambio climático. Rev. Mex. Cienc. Agríc. 2(Pub. Esp.):223-237.

Ruiz, C. J. A.; Medina, G. G.; Manríquez, O. J. D. y Ramírez, D. J. L. 2010. Evaluación de la vulnerabilidad y propuestas de medidas de adaptación a nivel regional de algunos cultivos básicos y frutales ante escenarios de cambio climático. Informe Final de Proyecto INIFAPINE. Guadalajara, Jal. 108 p. 
Ruiz, C. J. A.; Medina, G. G.; Rodríguez, M. V. M.; Sánchez, G. J. J.; Villavicencio, G. R.; Durán, P. N.; Grageda, G. J. y García, R. J. E. 2016. Regionalización del cambio climático en México. Rev. Mex. Cienc. Agríc. 13(Pub. Esp.):2451-2464.

Ruiz, C. J. A.; Ramírez, D. J. L.; Flores, M. F. J. y Sánchez, G. J. J. 2000. Cambio climático y efectos sobre las áreas potenciales para maíz en Jalisco, México. Rev. Fitotec. Mex. 23(2):183-194.

Salmi, T.; Maatta, A.; Anttila, P.; Ruoho, A. T. and Amnell, T. 2002. Detecting trends of annual values of atmospheric pollutants by the Mann-Kendall test and Sen's slope estimates- the Excel template application MAKESENS. Finnish Meteorological Institute, Air Quality Research. Publications on Air Quality No. 31. Report code FMI-AQ-31. 35 p.

Santillán, E. L. E.; Blanco, M. F.; Magallanes, Q. R.; García, H. J. L.; Cerano, P. J.; Delgadillo, R. O. y Valdez, C. R. D. 2011. Tendencias de temperaturas extremas en Zacatecas, México. Rev. Mex. Cienc. Agríc. 2(2):207-219.

Sen, P. K. 1968. Estimates of the regression coefficient based on Kendall's Tau. J. Am. Statistical Association. 63(324):1379-1389.

Snyder, R. L. 1985. Hand calculating degree-days. Agric. For. Meteorol. 35(4):353-358.

Van, V. D. P.; Edmonds, J.; Kainuma, M.; Riahi, K.; Thomson, A.; Hibbard, K.; Hurtt, G. C.; Kram, T; Krey, V.; Lamarque, J. F. and Masui, T. 2011. The representative concentration pathways: an overview. Climatic Change. 109(1):5-31.

Walton, D.; Meyerson, J. and Neelin, J. D. 2013. Accessing, downloading, and viewing CMIP5 Data. Earth System Grid Federation. 25 p.

Warmund, M. R. and Joan, K. 2005. A chilling model to estimate rest completion of erect blackberries. HortSci. 40(5):1259-1262.

Weinberger, J. H. 1950. Chilling requirement of peach varieties. Proc. Amer. Soc. Hort. Sci. 56:122-128.

$\mathrm{Yu}, \mathrm{H}$; Luedeling, E. and $\mathrm{Xu}$, J. 2010. Winter and spring warming result in delayed spring phenology on the Tibetan Plateau. PNAS. 107(XX):22151-22156.

Zarazúa, V. P.; Ruiz, C. J. A.; González, E. D. R.; Flores, L. E. H. y Ron, P. J. 2011a. Cambio climático y agroclimático para el ciclo otoño-invierno en la región Ciénega de Chapala. Rev. Mex. Cienc. Agríc. 2(Pub. Esp.):295-308.

Zarazúa, V. P.; Ruiz, C. J. A.; González, E. D. R.; Flores, L. H. E. y Ron, P. J. 2011b. Impactos del cambio climático sobre la agroclimatología del maíz en Ciénega de Chapala, Jalisco. Rev. Mex. Cienc. Agríc. 2(Pub. Esp.):351-363.

Zegbe, D. J. A.; Mena, C. J.; Medina, G. G.; Serna, P. A. y Echavarría, C. F. 2016. Cultivos frutícolas. Capítulo 3. In: Medina, G. G.; Zegbe, D. J. A.; Reveles, H. M.; Mena, C. J.; Reveles, T. L. y Echavarría, Ch. F. G. (Comp.). Libro técnico núm. 16. INIFAP-CIRNOC. Campo Experimental Zacatecas. 253-387 pp. 